a Departamento de Consulta Externa, Instituto Nacional de Cardiología Ignacio Chávez, México, D.F., México

b Departamento de Radiología e Imagen, Instituto Nacional de Cardiología Ignacio Chávez, México, D.F.,

México

'Departamento de Ecocardiografía, ABC Medical Center, I.A.P., México, D.F., México
* Autor para correspondencia. Consulta Externa, Instituto Nacional de Cardiología Ignacio Chávez, Juan Badiano, N. ${ }^{\circ}$ 1, Colonia Sección XVI, CP 14080 Tlalpan, México, D.F., México.

Correo electrónico: niesza2001@hotmail.com

(N. Espinola-Zavaleta).

http://dx.doi.org/10.1016/j.acmx.2015.07.003

\section{Recambio valvular tricuspídeo mínimamente invasivo utilizando oclusión percutánea endocava}

\author{
Minimally invasive tricuspid valve replacement \\ using percutaneous endocaval occlusion in \\ patients with previous cardiac surgery
}

\section{Introducción}

La disfunción de la válvula tricúspide puede ocurrir en válvulas estructuralmente normales o en válvulas con daño orgánico. La insuficiencia tricuspídea en pacientes con valvas normales suele ser secundaria a enfermedad valvular izquierda (es decir, insuficiencia tricuspídea funcional o secundaria) y es la principal causa de insuficiencia tricuspídea $^{1-4}$.

Únicamente el 35\% de los pacientes sometidos a cirugía valvular mitral estará libre de reoperación a 20 años, siendo la progresión de la valvulopatía tricuspídea la causante del $65 \%$ de dichas reintervenciones ${ }^{5,6}$.

Existen varios factores que incrementan la morbimortalidad de la cirugía de recambio valvular tricuspídeo, especialmente la presencia de cirugías cardiacas previas, lo cual se ve reflejado en la mayor incidencia de lesiones en estructuras cardiovasculares durante la disección (10\%), mayor sangrado postoperatorio (6.6\%), mayor incidencia de infecciones esternales (5-9.8\%). Con una mortalidad perioperatoria global asociada al cambio valvular tricuspídeo aislado que va desde el $11 \%$ hasta el $33 \%$ en algunas series $^{5-8}$.

El abordaje mínimamente invasivo para el recambio valvular tricuspídeo a través de minitoracotomía anterolateral derecha ha demostrado una disminución significativa de: mortalidad perioperatoria (2-6\%), sangrado (6\%), mediastinitis $(0 \%)$ y estancia hospitalaria (10 días). En los estudios que sustentan estos datos se ha observado un incremento en la incidencia de eventos cerebrovasculares $(9 \%)^{9,10}$.

\section{Métodos}

Estudiamos una serie de casos $(n=11)$, de todos los pacientes del Instituto Nacional de Cardiología «lgnacio Chávez» sometidos a recambio valvular tricuspídeo por minitoracotomía anterolateral derecha de enero del 2011 a enero del 2015. En todos los casos se utilizaron balones percutáneos para lograr el aislamiento de la aurícula derecha en lugar de realizar la disección de ambas cavas y la colocación de torniquetes o pinzas.

Las variables estudiadas fueron: mortalidad perioperatoria, sangrado posquirúrgico, infección profunda de la herida quirúrgica, tiempo de ventilación mecánica, tiempo de estancia en la unidad de cuidados intensivos y tiempo de estancia hospitalaria.

Técnica quirúrgica:

1. Se disecan arteria y vena femoral izquierdas así como vena yugular interna derecha.

2. Se realiza minitoracotomía anterolateral derecha de $4 \mathrm{~cm}$ en línea axilar anterior $4 .^{\circ}$ espacio intercostal.

3. Se administra heparina, $300 \mathrm{UI} / \mathrm{kg}$ vía intravenosa.

4. Se canulan arteria y vena femoral izquierdas y vena yugular interna derecha.

5. Por vía percutánea a nivel del vena femoral derecha se introducen 2 balones oclusores (balón de medición de dispositivo Amplatzer AGA-medical/St. Jude) uno en cava superior de $24 \mathrm{~mm}$ y otro en cava inferior de $34 \mathrm{~mm}$ de diámetro (fig. 1), lo más cercano a la unión cavoauricular posible, guiados por fluoroscopia.

6. Se inicia circulación extracorpórea en normotermia.

7. Se colapsa pulmón derecho.

8. Se insuflan balones oclusores endocava (fig. 2).

9. Se realiza pericardiotomía transversa a nivel de la aurícula derecha; la incisión involucra tanto el pericardio como la pared auricular, debido a las adherencias epicárdico-pericárdicas ocasionadas por la cirugía previa.

10. Se coloca separador de la aurícula derecha el cual se exterioriza hacia la pared torácica anterior a nivel de la línea medioclavicular por una incisión de $3 \mathrm{~mm}$.

11. Se realiza el cambio valvular tricuspídeo con 4 puntos separados de poliéster 00 en la porción septal del anillo y el resto del anillo con surgete continuo con polipropileno 00.

12. Se realiza atriorrafia derecha en 2 planos con polipropileno 3-0 involucrando el pericardio.

13. Se coloca marcapasos epicárdico temporal.

14. Se desinflan balones oclusores.

15. Se inicia destete de circulación extracorpórea hasta decanulación.

16. Se coloca drenaje blando $24 \mathrm{Fr}$ por contrabertura en pared costal. 


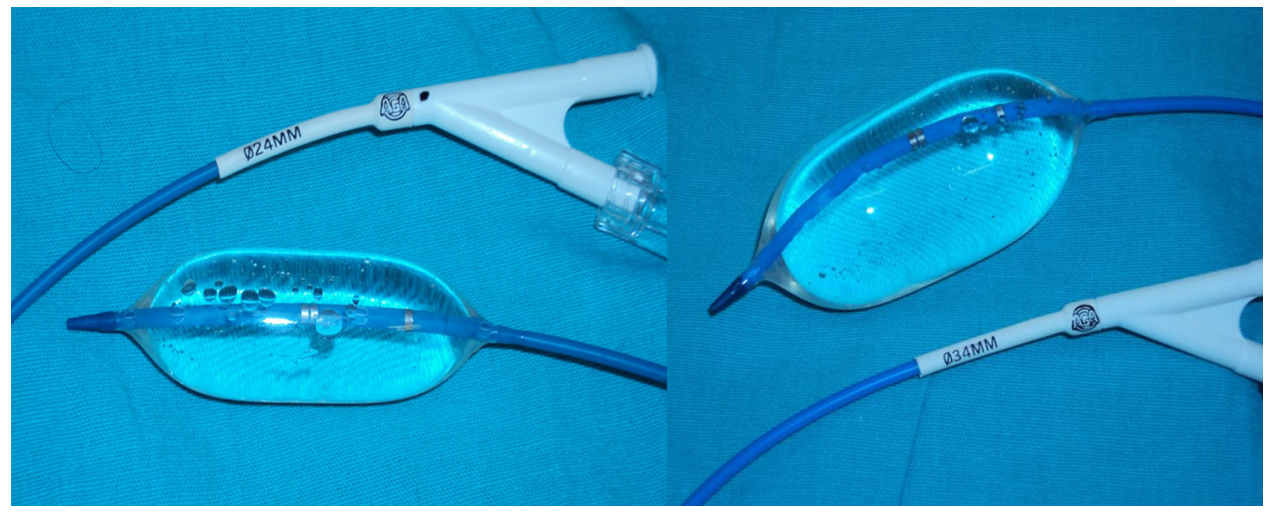

Figura 1 Balones percutáneos de medición de comunicación interauricular; $24 \mathrm{~mm}$ de diámetro para la cava superior y 34 mm para la cava inferior.

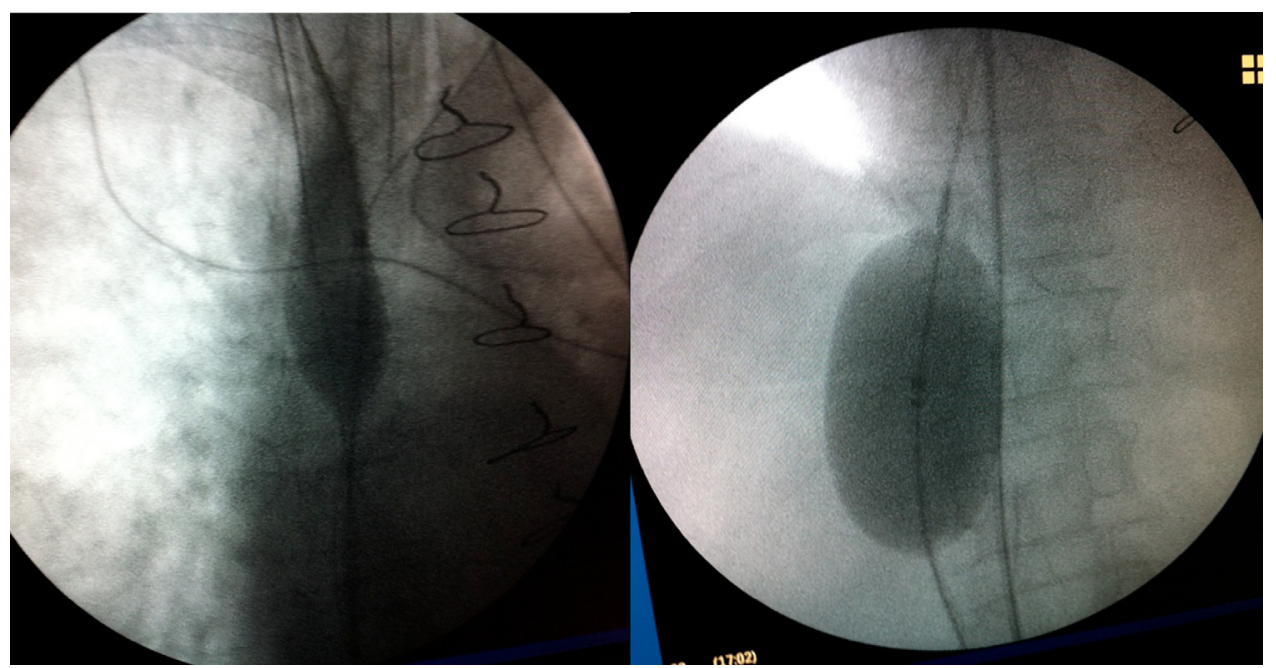

Figura 2 Imagen fluoroscópica en el momento del llenado de ambos balones para el aislamiento de la aurícula derecha.

\section{Resultados}

La población estudiada presentaba una edad promedio de 63 años, con un rango de 52-73 años. La totalidad de las pacientes eran del género femenino, el $9 \%$ (una paciente) con diagnóstico de diabetes mellitus, el 18\% (2 pacientes) eran portadoras de hipertensión arterial sistémica y el $9 \%$ (una paciente) con insuficiencia renal que requería sustitución de la función. La fracción de expulsión del ventrículo izquierdo en rangos del $45-73 \%$ con promedio del 61\%. Dentro de la clase funcional por escala de la New York Heart Association el $9 \%$ (una paciente) se encontraban en clase ।, el $27 \%$ ( 3 pacientes) en clase ॥ y el $63 \%$ (7 pacientes) en clase III. No hubo pacientes en clase funcional IV. El Euroscore ॥ promedio fue de $9.5 \%$ de mortalidad con un rango entre $3 \mathrm{y}$ $22 \%$ (tabla 1).

Dentro de las variables intra- y postoperatorias (tabla 2) obtuvimos un tiempo de derivación cardiopulmonar de 133 min (48-172), una paciente falleció $(9 \%)$ a las $96 \mathrm{~h}$ por disfunción ventricular derecha, el sangrado promedio en $24 \mathrm{~h}$ fue de $340 \mathrm{ml}$ (150-800). No se presentaron infecciones de herida quirúrgica. El tiempo de ventilación mecánica promedio fue de $45 \mathrm{~h}$ (12-72). El tiempo de estancia

\section{Tabla 1 Características basales de las pacientes}

\begin{tabular}{lc}
\hline Característica & Número \\
\hline Edad (años) & $63 \pm 10$ \\
Genero & \\
$\quad$ Femenino & $11(100 \%)$ \\
Diabetes mellitus & $1(9 \%)$ \\
Hipertensión & $2(18 \%)$ \\
Insuficiencia renal & $1(9 \%)$ \\
FEVI (\%) & $61(45-73)$ \\
Cirugía valvular previa & \\
CVM & $6(54 \%)$ \\
CVM+CVT & $3(27 \%)$ \\
CVM+CVT + CVAo & $2(18 \%)$ \\
Clase funcional (NYHA) & \\
I & $1(9 \%)$ \\
II & $3(27 \%)$ \\
III & $7(63 \%)$ \\
Euroscore II (\%) (promedio) & $9.5(3-22)$ \\
\hline
\end{tabular}

CVAo: cambio valvular aórtico; CVM: cambio valvular mitral; CVT: cambio valvular tricuspídeo; FEVI: fracción de expulsión del ventrículo izquierdo; NYHA: New York Heart Association. 
Tabla 2 Variables intra- y postoperatorias

\begin{tabular}{lc}
\hline Variables & Número \\
\hline Tiempo de DCP $(\mathrm{min})$ & $133(48-172)$ \\
Sangrado en $24 \mathrm{~h}(\mathrm{ml})$ & $340(150-800)$ \\
Infección de herida quirúrgica & 0 \\
Tiempo de ventilación mecánica $(\mathrm{h})$ & $45(12-72)$ \\
Tiempo de estancia en UCl $(\mathrm{h})$ & $48(36-96)$ \\
Tiempo de estancia hospitalaria (días) & $15(8-36)$ \\
\hline
\end{tabular}

DCP: derivación cardiopulmonar; UCI: Unidad de cuidados intensivos.

promedio en la unidad de cuidados intensivos fue de $48 \mathrm{~h}$ (36-96) y el tiempo de estancia intrahospitalaria promedio fue de 15 días (8-36).

\section{Discusión}

La técnica de recambio valvular tricuspídeo por abordaje de mínima invasión a través de minitoracotomía anterolateral derecha con oclusión percutánea endocava y en asistencia circulatoria es una técnica reproducible y segura, comparada con el abordaje tradicional por esternotomía media. En 2011 el grupo Italiano de Sansone ${ }^{11}$, describió el uso de catéteres urinarios de Foley (12-16 Fr.) para aislar ambas venas cavas, colocándolas bajo visión directa una vez realizada la auriculotomía derecha, lo cual aumenta el riesgo de entrada masiva de aire al sistema de derivación cardiopulmonar. Posteriormente este mismo autor en $2012^{12}$ reportó la técnica de oclusión percutánea endocava con el balón oclusor Equalizer $^{T M} 7 \mathrm{Fr}$ en 8 pacientes, todos con cirugía cardiaca previa, utilizando la ecocardiografía transesofágica para el posicionamiento de los balones oclusores en ambas cavas; este método es muy similar al que describimos en el presente trabajo salvo el tipo de balón utilizado y el apoyo fluoroscópico para realizar el posicionamiento de los balones, sin embargo ambos estudios reportaron buenos resultados. En $2014^{13}$ el grupo chino del Dr. Zhaolei reportó la oclusión de ambas venas cavas utilizando tubos endotraqueales que se colocan de manera directa a través de la pared de la aurícula derecha; esta técnica tiene la desventaja de reducir de forma importante el espacio de trabajo ya que ambas cánulas (10 $\mathrm{mm}$ de diámetro externo de cada una) se exteriorizan a través de la toracotomía, lo cual incrementa ligeramente la dificultad de la técnica, sin embargo reportan buenos resultados.

El presente estudio tiene como objetivo el presentar una novedosa técnica para realizar la oclusión percutánea endoluminal de ambas venas cavas, en pacientes reoperados que requieran cirugía tricuspídea, con la finalidad de disminuir el riesgo de lesión de estructuras vasculares durante la disección.

\section{Financiación}

Ninguno.

\section{Conflicto de intereses}

Los autores declaran no tener ningún conflicto de Intereses.

\section{Bibliografía}

1. Cohn LH. Tricuspid regurgitation secondary to mitral valve disease: When and how to repair. J Card Surg. 1994;9: 237-41.

2. Ratnatunga CP, Edwards M, Dore CJ, et al. Tricuspid valve replacement: UK Heart Valve Registry mid-term results comparing mechanical and biological prostheses. Ann Thorac Surg. 1998;66:1940-7.

3. Nath J, Foster E, Heidenreich PA. Impact of tricuspid regurgitation on long-term survival. J Am Coll Cardiol. 2004;43: 405-9.

4. Singh S, Tang G, Maganti M, et al. Midterm outcomes of tricuspid valve repair versus replacement for organic tricuspid disease. Ann Thorac Surg. 2006;82:1735-41.

5. Bernal J, Morales D, Revuelta C, et al. Reoperations after tricuspid valve repair. J Thorac Cardiovasc Surg. 2005;130: 498-503.

6. Elahi M, Dhannapuneni R, Firmin R, et al. Direct complications of repeat median sternotomy in sdults. Asian Cardiovasc Thorac Ann. 2005;13:135-8.

7. Park C, Park P, Sung K, et al. Early and midterm outcomes for tricuspid valve surgery after left-sided valve surgery. Ann Thorac Surg. 2009;88:1216-23.

8. Jones J, O'kane H, Gladstone D, et al. Repeat heart valve surgery: Risk factors for operative mortality. J Thorac Cardiovasc Surg. 2001;122:913-8.

9. Lee T, Desai B, Glower D. Results of 141 consecutive minimally invasive tricuspid valve operations: An 11-year experience. Ann Thorac Surg. 2009;88:1845-50.

10. Kypson A, Glower D. Minimally invasive tricuspid operation using port access. Ann Thorac Surg. 2002;74:43-5.

11. Sansone F, del Ponte S, Zingarelli E, et al. Internal snaring of the caval veins by Foley catheters in case of reoperation via right thoracotomy. Interact Cardiovasc Thorac Surg. 2011;13:370-2.

12. Sansone F, Barbero C, Rinaldi M. Occlusion of both caval veins by an endovascular occluder. Heart Lung Circ. 2012;21:275-7.

13. Jiang Z, Mei J, Ding F, et al. Application of endovascular occlusion of both caval veins in minimally invasive isolated redo tricuspid surgery through right thoracotomy. Heart Lung Circ. 2014;23:186-9.

Miguel Corona*, Javier Sagahón, Iván Hernández, Alejandra Iturriaga, Humberto Martínez y Valentín Herrera

Departamento de Cirugía Cardiovascular, Instituto Nacional de Cardiología Ignacio Chávez, México, D.F, México

* Autor para correspondencia. Av. Moliere 342, departamento 16, Colonia Los Morales III Sección, Delegación Miguel Hidalgo, México, Distrito Federal, C.P. 11540.

Teléfonos: (55) 32-22-07-17 y 52-82-17-41.

Correo electrónico: coronaperezgrovas@gmail.com (M. Corona).

http://dx.doi.org/10.1016/j.acmx.2015.08.004 